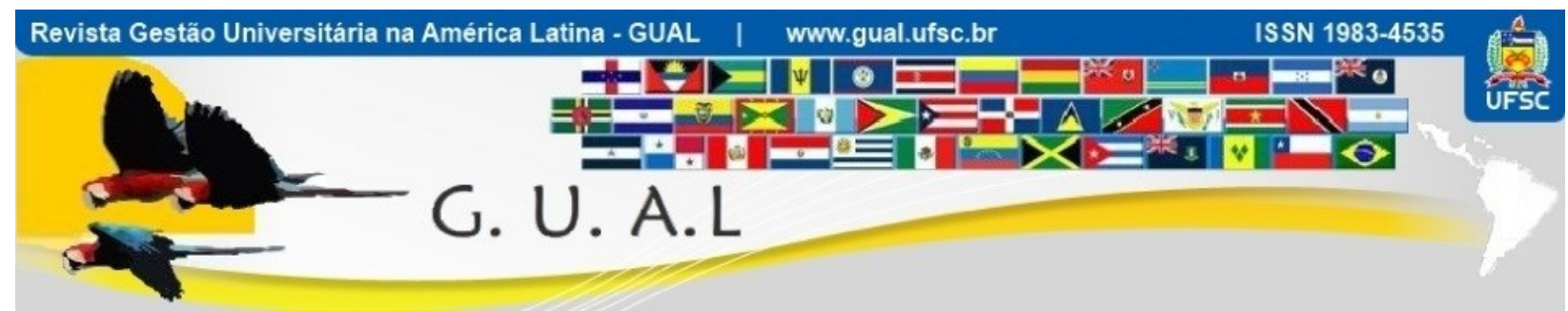

DOI: http://dx.doi.org/10.5007/1983-4535.2016v9n2p95

\title{
ESTILO DE APRENDIZAGEM DOS ALUNOS DO CURSO DE CIÊNCIAS CONTÁBEIS
}

\section{LEARNING STYLE OF ACCOUNTING SCIENCES STUDENTS}

\author{
Raimundo Nonato Lima Filho, Doutor \\ Universidade do Estado da Bahia - UNEB \\ rnlfilho@gmail.com \\ Eliane da Silva Bezerra, Graduada \\ Universidade do Estado da Bahia - UNEB \\ eliane.bahia@sitiobarreiras.com.br
}

Thiago Bruno de Jesus Silva, Mestrando Fundação Universidade Regional de Blumenau - FURB thiagobruno.silva@yahoo.com.br

Recebido em 05/julho/2014

Aprovado em 18/janeiro/2016

Sistema de Avaliação: Double Blind Review

Esta obra está sob uma Licença Creative Commons Atribuição-Uso. 


\title{
RESUMO
}

Esta pesquisa objetiva identificar o estilo de aprendizagem predominante em alunos do Curso de Graduação em Ciências Contábeis nas modalidades de ensino presencial e EAD em instituições públicas e privadas do Estado da Bahia. Para alcançar os objetivos propostos foi utilizado o Learning Style Inventory (LSI). Os participantes do estudo constituíram-se de 459 alunos do curso de Ciências Contábeis. $\mathrm{Na}$ análise estatística dos dados, constatou-se haver predominância do estilo de aprendizagem assimilador, representado por pessoas que aprendem por meio da observação reflexiva e conceituação abstrata. Sendo assim, este estudo contribui para melhoria do ensino-aprendizagem em Contabilidade, uma vez que possibilita às instituições envolvidas conhecer o estilo de aprendizagem do aluno e assim, criar mecanismos cognitivos que facilitem o processamento das informações ao mesmo tempo em que permite direcionar sua metodologia levando em consideração o estilo predominante e o estímulo dos estilos secundários.

Palavras chaves: Ciências Contábeis. Estilo de aprendizagem. Modalidades de ensino. Modelo Kolb. Ensino presencial. Ensino EAD.

\begin{abstract}
This study attempts to identify the predominant learning style of accounting undergraduate course students in the modalities of classroom learning and distance education in both public and private institutions in the state of Bahia. To achieve the proposed objectives the Learning Style Inventory (LSI) was used. The participants in this study consisted of 459 students from the accounting course. In the statistical analysis, it was found that there was a predominance of assimilating learning style, represented by people who learn through reflective observation and abstract conceptualization. Thus, this study contributes to improving teaching and learning in accounting, as it enables the involved institutions to get to know the learning style of the students. Therefore, to create cognitive mechanisms that facilitate the processing of information while allowing to direct their leading methodology taking into account the predominant style and the stimulation of secondary styles.
\end{abstract}

Keywords: Accounting. Learning style. Teaching modalities. Kolb Model. Classroom teaching. EAD education. 


\section{INTRODUÇÃO}

Durante o processo de ensino-aprendizagem nasce a grande oportunidade de desenvolver a autonomia na construção do conhecimento e capacidade de aprender. Os alunos têm diferentes maneiras de aprender e manipulam as informações por métodos distintos. Fatores como personalidade, característica, níveis de dificuldade das tarefas e da aprendizagem estão fortemente envolvidos.

As recomendações da Accounting Education Change Commission (AECC, 1990), do American Institute of Certified Public Accountants (AICPA, 2000) e Institute of Management Accountants, (IMA, 2008) apontam para a necessidade de uma formação em sala de aula que conduza os estudantes (de Contabilidade) a adquirirem atributos e habilidades de aprendizagem permanente. Oliveira, Raffaelli, Colauto \& Casa Nova (2013) explicam que as modificações ocorridas no mercado de trabalho têm pressionado as instituições do ensino superior a modificarem o ensino em Ciências Contábeis no Brasil, uma vez que o processo de convergência da norma contábil brasileira às normas internacionais.

Para Cerqueira (2000), o estilo de aprendizagem está voltado para a organização e o controle das estratégias de aprendizagem. Desta forma, o estilo de aprendizagem está relacionado com a maneira pela qual as pessoas interagem com as condições que lhes são impostas, abrangendo aspectos cognitivos, afetivos, físicos e ambientais. Sendo uma preferência característica e dominante que retrata a forma como recebem e processam as informações de forma que possam desenvolver habilidades cognitivas. Ku, Shing e Hung (2014) afirmam que identificar os estilos de aprendizagem pode facilitar o ensino e aprendizagem, sendo uma questão crucial na educação.

David Kolb (1984) descreve a aprendizagem como um processo de ressignificação da realidade, no qual ocorre por meio da relação dialética entre as experiências anteriores e a reflexão relacionada a novas informações adquiridas pelo sujeito. Inspirado na Teoria Cognitiva, sobretudo nas premissas de formação histórico-cultural de Vygotsky (1987), Kolb desenvolve o ciclo de aprendizagem experiencial. O ciclo é composto por quatro modelos de aprendizagem. O primeiro modelo intitulada Experiência Concreta (EC), refere-se à capacidade de aprender por meio dos sentidos e dos sentimentos. O segundo modelo é o de Observação Reflexiva (OR) e caracteriza as estruturas cognitivas voltadas para o interior e para a reflexão a partir da observação. O terceiro modelo refere-se à conceituação abstrata (CA), relacionando-se à formação de conceitos abstratos, generalização sobre elementos e 
características da experiência, compreendendo a lógica das ideias. E por fim, o quarto modelo diz respeito à Experiência Ativa (EA) voltada para aspectos externos da ação relacionada com relações interpessoais em que se aprende fazendo, praticando (Manolis; Burns; Assudani \& Chinta, 2013).

Mcchlery \& Visser (2009) afirmam que os estilos de aprendizagem tornam-se importante para o professor visando compreender o perfil dos alunos para determinar a escolha da estratégia de ensino mais adequada para obtenção de maior aproveitamento do processo de ensino e aprendizagem. Na visão de que o aluno tem preferências na maneira de absorver as informações, instiga a necessidade do professor, estimula a importância do docente em modificar os seus métodos de ensino. Quando o estilo de aprendizagem é incompatível com o estilo de ensino, o desempenho acadêmico pode ser afetado.

As formas em que os alunos aprendem no ensino superior tem sido o foco de muita investigação visando identificar e categorizar os estilos de aprendizagem dos alunos (Richardson; Dellaportas \& Perera, 2013). Desta forma, a questão que conduz e orienta esta investigação é: - Qual estilo de aprendizagem predominante nos alunos de graduação em Ciências Contábeis na modalidade de ensino EAD e presencial? Para responder a esta questão, fez-se opção pelo LSI desenvolvido pelo pesquisador de comportamento organizacional David Kolb (1984). Assim, este estudo tem por objetivo de identificar o estilo de aprendizagem predominante em alunos do Curso de Graduação em Ciências Contábeis nas modalidades de ensino presencial e EAD em instituição pública e privada do Estado da Bahia.

A pesquisa se justifica por entender que alunos têm um estilo próprio de aprendizagem e identificá-los possibilitará o desenvolvimento de métodos de ensino que melhor atendam as necessidades de aprendizagem bem como, a percepção de como se apresenta a habilidade cognitiva dos alunos do curso de Ciências Contábeis tanto no âmbito presencial como EAD.

\section{REFERENCIAL TEÓRICO}

\subsection{PROCESSO DE APRENDIZAGEM}

O processo de aprendizagem pode ser visto em diversas formas, não apenas no educacional, porém, é neste que ocorre a interações entre os elementos: "discente" enquanto aprendiz, "instituição" como suporte de apoio, "assunto" aqui identificado como conteúdo curricular e "docente" que é a pessoa que fornecerá o suporte necessário à viabilização do aprendizado. Segundo Tanner \& Morgan (2007) a aprendizagem dura toda a vida, enquanto 
vivemos estamos em processo de aprendizagem e esse processo ocorre de maneira pessoal e natural.

No que concerne às atividades docentes, Freire (2006) diz que o professor precisa ter em mente clareza dos conteúdos ministrados, para que consiga, em sua fala, envolver o aluno e fazê-lo viajar sobre o intimo dos seus pensamentos. É preciso o uso de estratégias que motive o discente a participar das aulas.

Dessa forma, as diferenças de aprendizagem podem estar relacionadas com a maneira de aprender dos indivíduos, às estratégias utilizadas destacando a memória e o tempo de reação do individuo.

\subsection{ESTILOS DE APRENDIZAGEM}

Estilo de aprendizagem é entendido por Silva (2006), como uma espécie de enfoque pessoal através do qual os indivíduos respondem as diversas situações de aprendizagem e a maneira como a pessoa pensa. Trata da abordagem sistêmica do sujeito com a organização e o processamento da informação durante a construção do conhecimento. (Manolis et al, 2013) descrevem o estilo de aprendizagem de aprendizagem, como definida como a orientação de um indivíduo à aprendizagem que permite aprender melhor. $\mathrm{O}$ estilo de aprendizagem de um indivíduo refere-se à forma como recebe, interage e responde aos ambientes de aprendizagem.

David Kolb (1976) iniciou seus estudos sobre estilos de aprendizagem a partir de 1971 e desenvolveu uma linha de pesquisa que tem como alvo principal estudantes universitários. Seu instrumento evidencia a forma como o conhecimento é processado, no sentido de entender como se aprende e assimila as diversas informações, de que maneira os problemas são solucionados e como as decisões são tomadas, onde o aluno passa por quatro estágios básicos de aprendizagem que deu o nome de ciclo de aprendizagem

Esse processo cíclico funciona como uma espécie de feedback, que se baseia na nova ação e avaliação das consequências e ações. As pessoas podem passar pelo ciclo diversas vezes, esse movimento é classificado como "Espiral de Ciclo de Aprendizagem constituído por quatro momentos principais: planejar, agir, observar e refletir" (Kolb, 1984). 


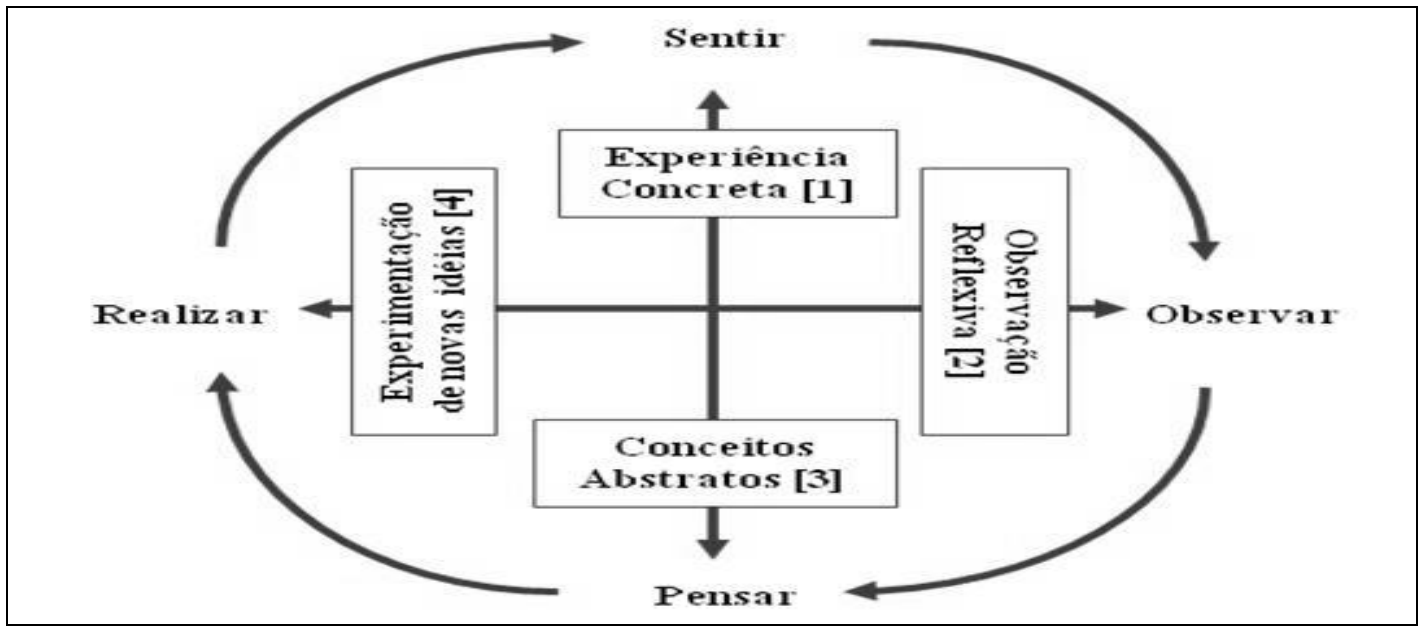

Figura 1 Compreensão dos fenômenos.

Fonte: David Kolb (1985).

Experiência Concreta (EC) envolve os sentidos e os sentimentos, aqui o individuo confia mais em suas emoções do que no enfoque sistêmico do problema. Observação Reflexiva (OR) envolve a observação e audição, os alunos aprendem observando, veem as coisas cuidadosamente. Conceituação Abstrata (CA), o individuo portador desse estilo aprende pensando, entende o uso da lógica e das ideias tende a desenvolver e analisar teorias para resolver problemas. Experimentação Ativa (EA), o estudante aprende fazendo, possui habilidades que facilitam o cumprimento de tarefas que envolvem riscos, por valorizar os resultados decorrentes de sua influência.

Para Kolb (1984), a melhor maneira de desenvolver o conhecimento é por meio das experiências vividas dentro do ambiente social. Uma aprendizagem para ser bem sucedida demanda do aluno a combinação dessas etapas, podendo evoluir para padrões estáveis e duradouros que caracterizam a individualidade de cada um.

O estilo acomodador caracteriza-se pelo fazer e sentir. As pessoas que o possuem, desenvolvem suas preferências baseadas na experimentação ativa e concreta. $\mathrm{O}$ assimilador apresenta pessoas que aprendem por observação, reflexão e conceituação abstrata, possuem habilidades para criar modelos abstratos e teóricos. Aqueles com estilo convergente possuem capacidade de desenvolver conceitos abstratos e experiência ativa (Turesky \& Gallagher, 2011).

Mendes (2012) analisou o estilo de aprendizagem em estudantes de Contabilidade nos Estados Unidos e na Noruega. Foi identificado o estilo convergente como predominante em alunos nos EUA e um estilo assimilador nos alunos da Noruega, concluindo que essa diferença afeta o cenário de educação contábil internacional no que concerne o 
desenvolvimento de programas educacionais ou de treinamento na tentativa de atender todos os estilos existentes.

Nogueira, Espejo, Reis \& Voese (2012), verificaram se o desempenho dos alunos de educação a distância nas disciplinas de Contabilidade Geral, Gerencial e no módulo de Contabilidade é diferente de acordo com seu estilo de aprendizagem. A pesquisa foi realizada com 109 alunos de um curso de educação a distância e utilizou como variável independente o estilo de aprendizagem (verificado pelo LSI de Kolb) e como variável dependente a nota nas disciplinas de Contabilidade Geral, Gerencial e do módulo de Contabilidade (média entre a nota de contabilidade geral e gerencial). Foram realizados testes de confiabilidade (Alpha de Cronbach) para o instrumento LSI de Kolb e testes de normalidade dos dados. Além disso, procedeu-se a análises estatísticas descritivas e testes de diferenças de médias (Kruskall-wallis e ANOVA) para responder a questão de pesquisa. Os resultados evidenciam que a maior parte dos alunos é do estilo Assimilador (44\%) e Divergente (34\%). E indicam que não foi possível constatar que os estilos de aprendizagem proporcionassem diferenças no desempenho dos alunos.

Richardson, Dellaportas e Perera (2013) objetivaram avaliar os benefícios derivados da aplicação de dispositivos móveis como parte da experiência de aprendizagem on-line dos alunos e na medida em que os benefícios percebidos estão ligados a estudante de estilos de aprendizagem. Um pequeno grupo de estudantes matriculados em uma pós-graduação on-line em contabilidade receberam iPods, como parte de seus materiais de aprendizagem. Os resultados indicaram que a principal vantagem de iPods reside na sua portabilidade.

Santos et al. (2013) objetivaram evidenciar o estilo de aprendizagem predominante dos alunos do Curso de Ciências Contábeis da Universidade Federal de Mato Grosso do Sul, Campus do Pantanal. Trata-se de um estudo descritivo, formal, ex post facto, em condições de campo, transversal, de rotina real, estatístico, utilizando-se de coleta de dados com questionário previamente formulado, aplicado aos acadêmicos objetos desta pesquisa. A pesquisa que foi realizada durante o mês de janeiro de 2013 e constituiu-se de 75 acadêmicos dos 144 matriculados e com frequência no curso. Como resultados obtidos, verificou-se a predominância nos acadêmicos do estilo assimilador, em 54,66\% dos entrevistados. O estilo assimilador revela que as pessoas consideram que é mais importante que uma teoria tenha um sentido lógico do que um valor prático. Dessa forma, conhecendo e explorando o estilo de aprendizagem do acadêmico em Ciências Contábeis, obtém-se um fator importante para 
contribuir para a formação de um profisssional preparado para os desafios da profíssão e o sucesso empresarial.

Oliveira et al. (2014) tiveram o objetivo geral analisar se existe relação das variáveis idade, gênero e estilo de aprendizagem na percepção de estudante de contabilidade sobre o emprego de estratégias lúdicas. A coleta de dados ocorreu por meio de instrumento de pesquisa elaborado pelos autores a partir do inventário de Kolb (1984) e validado com Alfa de Cronbach de 0,873. A amostra, intencional, foi composta por 206 estudantes matriculados no curso de Ciências Contábeis em uma universidade federal do sul do Brasil. Os resultados apontam indícios de que os estilos de aprendizagem estão relacionados à percepção dos estudantes quanto ao uso de estratégias lúdicas em sala de aula. Quanto às variáveis idade e gênero, não se verificou evidências significativa da relação. Os resultados confirmam a importância de se considerar os estilos de aprendizagem ao se planejar as estratégias de ensino a serem adotadas.

A identificação do estilo de aprendizagem predominante nos alunos permite contribuir para as ações de transformações necessárias ao desenvolvimento cognitivo. Uma vez que, o processo de aprendizagem ocorre quando alunos e professores compreendem as relações envolvidas no processo de aquisição de conhecimento. Segundo McCarth (2010), estudos relacionados aos estilos de aprendizagem se tornaram uma preocupação com o intuito de saber como o estudante aprende, como se dá o processo de aprendizado, as abordagens de personalidade, processamento de informações, a interação social e as preferências relacionadas às instruções.

\subsection{ENSINO PRESENCIAL E ENSINO A DISTÂNCIA (EAD)}

A educação à distância tem como característica marcante o autoaprendizado, através dele o aluno é impulsionado a buscar o conhecimento de maneira independente e colaborativa. A modalidade de ensino presencial faz com que o contato físico seja estabelecido, o que possibilita um acompanhamento mais preciso do aluno em relação às matérias e ao professor.

Para Moore e Kearsley (2007, p. 2), a "Educação a distância é o aprendizado planejado que ocorre normalmente em um lugar diferente do local de ensino, exigindo técnicas especiais de criação do curso e de instrução comunicadas por várias tecnologias e disposições organizacionais e administrativas especiais". É uma modalidade de ensino na qual a mediação didático-pedagógica ocorre com a utilização de meios e tecnologias de 
informação e comunicação, onde estudantes professores desenvolvem atividades educativas em lugares e tempos diferentes.

Mill, Ribeiro e Oliveira (2010, p. 23), explicam as diferenças entre a EaD e a educação presencial, pois "O trabalho docente na Educação a Distância (EaD) é extremamente fragmentado, e cada parte das atividades que compõem o trabalho docente virtual é atribuída a um trabalhador diferente ou a um grupo deles".

Cornachione Jr, Casa Nova e Trombetta (2007), concluíram que os atributos que influenciam na decisão de um aluno matricular-se em um curso on-line são principalmente a instituição (renome, credibilidade, etc.) e o conteúdo do curso. Esse destaque por parte dos alunos em relação à instituição é justificado, pois uma das grandes preocupações dos discentes é saber se, depois de concluir o curso, o mesmo será reconhecido pelo MEC.

Reis et al. (2013) desenvolveram uma pesquisa com o objetivo de comparar o processo de ensino-aprendizagem nas metodologias de ensino presencial e a distância em cursos de Ciências Contábeis nas disciplinas de Contabilidade de Custos e Contabilidade Gerencial. Com a utilização da técnica de análise de conteúdo, constataram haver pontos divergentes nas duas modalidades de ensino, em especial no que diz respeito ao processo de avaliação de desempenho e da utilização de recursos pedagógicos. Os docentes da modalidade presencial possuem melhor desempenho em relação aos da modalidade a distancia nas referidas disciplinas.

\section{PROCEDIMENTOS METODOLÓGICOS}

Visando alcançar o objetivo deste estudo, a técnica utilizada foi a aplicação de questionário, os dados foram obtidos por meio da aplicação do LSI de Kolb, sendo caracterizada como pesquisa descritiva de natureza aplicada e formal. Caracteriza-se como um estudo em um ambiente de campo onde o pesquisador questiona os sujeitos e coleta as respostas por meio de entrevistas ou aplicação de questionário. Martins e Cox (2013) inferem que o estudo quantitativo possibilita organizar, caracterizar e interpretar dados numéricos coletados, por meio da aplicação de métodos e técnicas estatísticas. Os dados foram disponibilizados no ambiente de criação de questionários virtual.

A pesquisa foi realizada em alunos do curso de Ciências Contábeis em todo Estado da Bahia. O estudo envolveu 73 instituições de ensino, dessas, 20 são instituições de ensino público e 53 de ensino particular. Quanto à modalidade, 53 são presenciais e $20 \mathrm{EAD}$. Os participantes responderam ao questionário usando grau de 1 a 4, completando ao final de cada 
sentença de acordo com o que considera como sua maneira de aprender, ordenando as frases de cada conjunto de 1 a 4 .

Kolb desenvolveu seu LearningStyle Inventory (LSI) ou Inventário dos Estilos de Aprendizagem (IEA), que é o instrumento visando identificar os estilos de aprendizagem dos alunos pesquisados. O LSI de Kolb é um questionário com doze sentenças com quatro respostas para cada pergunta. As respostas são realizadas de forma ordinal, sendo que o aluno deve atribuir o $n^{\circ} 4$ às respostas que ele mais se identifica e $n^{\circ} 1$ para aquela que ele menos se identifica no momento da aprendizagem. Demonstra-se a seguir um exemplo de resposta, conforme no quadro 1.

\begin{tabular}{|l|l|l|l|l|l|l|l|l|}
\hline Sentença & A & $\mathrm{N}^{\circ}$ & $\mathrm{B}$ & $\mathrm{N}^{\mathrm{o}}$ & $\mathrm{C}$ & $\mathrm{N}^{\circ}$ & $\mathrm{D}$ & $\mathrm{N}^{\mathrm{o}}$ \\
\hline Aprendo & Sentindo & 1 & Observando & 2 & Fazendo & 3 & Pensando & 4 \\
\hline
\end{tabular}

Quadro 1 Modelo de Preenchimento das Sentenças.

Fonte: Adaptado de Cerqueira (2000).

Por meio do quadro 1, percebe-se que o respondente aprende com maior facilidade Fazendo, em segundo lugar, Pensando, em seguida, Observando, e a última maneira que poderia aprender algum conteúdo seria Sentindo.

\section{ANÁLISES DOS DADOS}

Nesse tópico será abordado a análise dos dados, evidenciando a forma como os mesmos foram tratados; em números absolutos, percentuais e quantidade de alunos de acordo com o Estilo de Aprendizagem. A análise é feita individualmente e por grupo e os dados serão ordenados de forma que seja possível responder a questão da pesquisa e atender o objetivo estabelecido.

A pesquisa abrangeu 459 respondentes, onde 349 são alunos que estudam na modalidade de ensino presencial e 110 na modalidade EAD. Destes, 190 são de instituições públicas e 269 de instituições particulares.

Em relação à classificação por gênero, verifica-se que $46 \%$ são alunos do sexo masculino e $54 \%$ do sexo feminino. 
Tabela 1 Distribuição do Gênero entre as Variáveis.

\begin{tabular}{|c|c|c|c|c|}
\hline Gênero & Instituição & Modalidade de Ensino & Número & $\%$ \\
\hline \multirow{4}{*}{ Feminino } & \multirow{2}{*}{ Pública } & EAD & 0 & $0 \%$ \\
\hline & & Presencial & 101 & $22 \%$ \\
\hline & \multirow{2}{*}{ Privada } & EAD & 50 & $11 \%$ \\
\hline & & Presencial & 98 & $21 \%$ \\
\hline \multirow{4}{*}{ Masculino } & \multirow{2}{*}{ Pública } & EAD & 0 & $0 \%$ \\
\hline & & Presencial & 89 & $19 \%$ \\
\hline & \multirow{2}{*}{ Privada } & EAD & 55 & $12 \%$ \\
\hline & & Presencial & 66 & $15 \%$ \\
\hline \multicolumn{3}{|l|}{ Total } & 459 & $100 \%$ \\
\hline
\end{tabular}

Fonte: Elaboração própria (2015).

Os dados (Tabela 1) mostram que a maioria dos participantes é do sexo feminino (43\%) da modalidade presencial de ensino contra 34\% do sexo masculino. Enquanto no ensino semipresencial (EAD) $11 \%$ feminino e $12 \%$ masculino.

O resultado apresentado está em consonância com os encontrados por Batista (2013), em sua pesquisa envolvendo estilo de aprendizagem e metodologia de ensino em alunos do curso de Ciências Contábeis da Universidade do Estado da Bahia campus VII, o qual identificou haver predominância do gênero feminino $(57,52 \%)$ em detrimento do masculino $(42,48 \%)$.

\subsubsection{Análise das Preferências de Aprendizagem}

Para Nogueira (2009), alguns alunos percebem a informação por meio da experiência concreta, visualizando situações, ouvindo, tocando ou experimentando os fatos. Outros, por intermédio de experiência ativa, utilizando conceitos mentais; existem aqueles que utilizam a experiência abstrata desenvolvendo pensamento.

Tabela 2 Frequência das Preferências de Aprendizagem.

\begin{tabular}{l|c|c}
\hline \multicolumn{1}{c|}{ Preferência } & Frequência Absoluta & Frequência Relativa \\
\hline Experiência Concreta (EC) & 120 & $26,14 \%$ \\
\hline Conceituação Abstrata (CA) & 70 & $15,25 \%$ \\
\hline Observação Reflexiva (OR) & 261 & $56,86 \%$ \\
\hline Experimentação Ativa (EA) & 8 & $1,74 \%$ \\
\hline Total & $\mathbf{4 5 9}$ & $\mathbf{1 0 0 , 0 0 \%}$ \\
\hline
\end{tabular}

Fonte: Elaboração própria (2015) 
As preferências de aprendizagem identificadas estão distribuídas na Tabela 2. Por meio da análise, é possível observar que existe predominância da Observação Reflexiva (OR) correspondendo a 56,86\% dos respondentes seguindo da Experiência Concreta (EC) 26,14\%, a Experimentação Ativa (EA) representa o menor número de respondentes com $1,74 \%$ das preferências dos alunos.

Logo, os alunos aprendem por meio da Observação Reflexiva (OR) representada pela palavra "Observar" e Experimentação Ativa (EA) definida pela palavra "Fazer”. Gostam de aprendem observando, fazendo e pensando, buscando contato direto com o objeto de estudo.

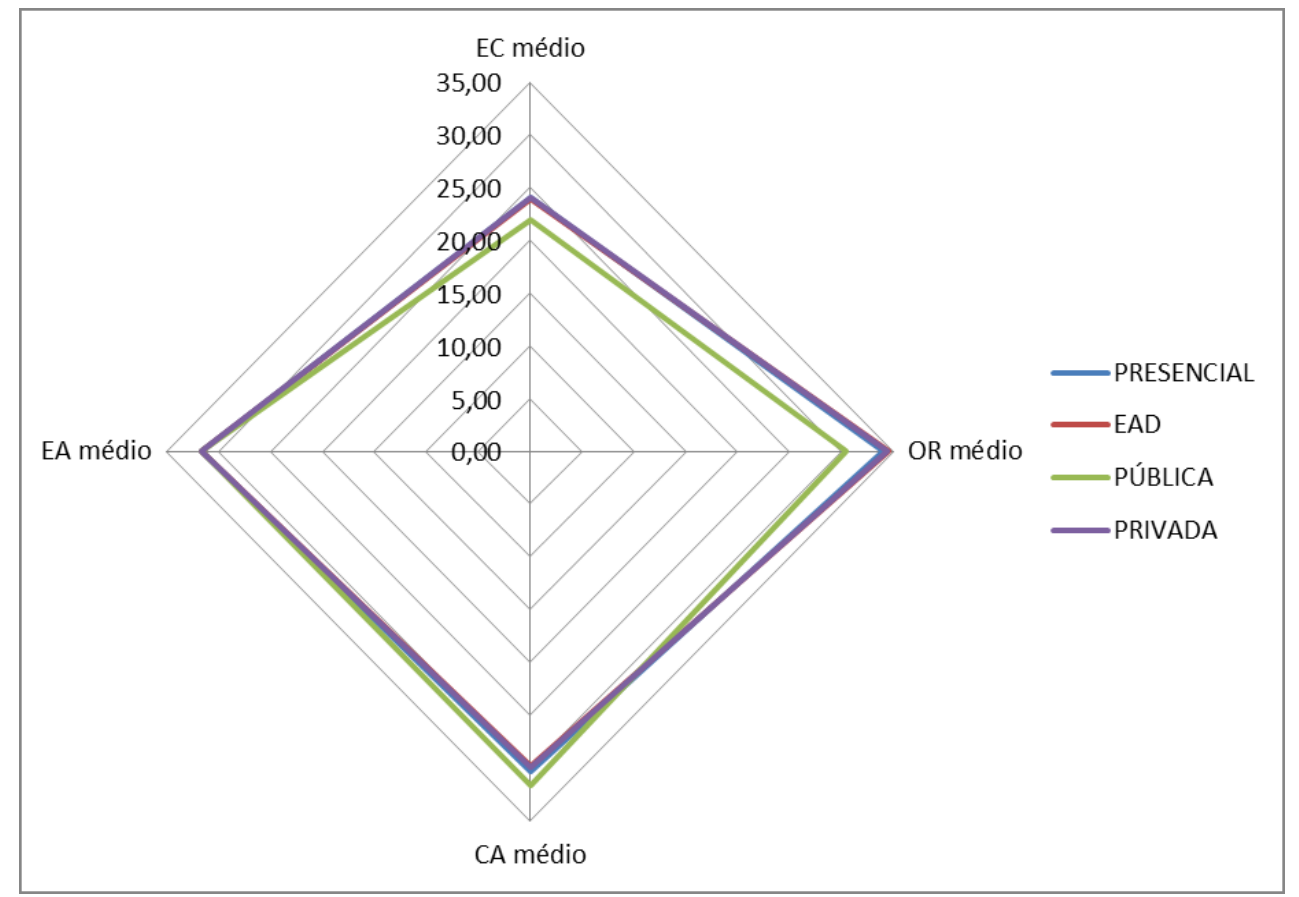

Gráfico 1 Distribuição das Médias entre as Preferências de Aprendizagem. Fonte: Elaboração própria (2015).

O Gráfico 1 apresenta a distribuição da média geral entre as variáveis (tipo de instituição e modalidade de ensino). Depreende-se das informações expostas que, existe equilíbrio, ou seja, os alunos podem adaptar-se a qualquer preferência ou até desenvolver as quatro ao mesmo tempo. 
Tabela 3 Estilo de Aprendizagem por Tipo de Instituição.

\begin{tabular}{l|c|c|c|c|c|}
\hline & \multicolumn{3}{|c}{ TIPO DE INSTITUIÇÃO } \\
\hline ESTILO & PÚBLICA & & \multicolumn{3}{c}{ PRIVADA } \\
\hline Convergente & Frequência & $\%$ & Frequência & $\%$ & \\
\hline Divergente & 15 & $8 \%$ & 23 & $9 \%$ \\
\hline Acomodador & 45 & $24 \%$ & 73 & $27 \%$ \\
\hline Assimilador & 12 & $6 \%$ & 10 & $4 \%$ \\
\hline TOTAL & 118 & $62 \%$ & 163 & $61 \%$ & \\
\hline
\end{tabular}

Fonte: Elaboração própria (2015).

Como explicito na Tabela 3, a maior parte dos alunos, tanto das instituições públicas quanto das privadas, apresentam o estilo de aprendizagem assimilador, (62\%) e (61\%), respectivamente. Isso significa que eles têm como característica principal a observação reflexiva $(\mathrm{OR})$ e conceituação abstrata $(\mathrm{CA})$, ou seja, gostam de aprender por meio de situações práticas.

Cox (2013) identificou resultados diferentes, quando pesquisou um grupo de alunos do curso de Enfermagem na University Central of Florida e identificou como estilo de aprendizagem predominante o acomodador. Essa diferença pode está relacionada ao tipo da amostra e o curso analisado. Essa preferência reflete o resultado de uma educação centrada na figura do professor. Kolb (1985) defende que os optantes por esse estilo, geralmente, são pessoas que confiam mais nos outros para conseguir informações do que em sua própria análise cognitiva. 


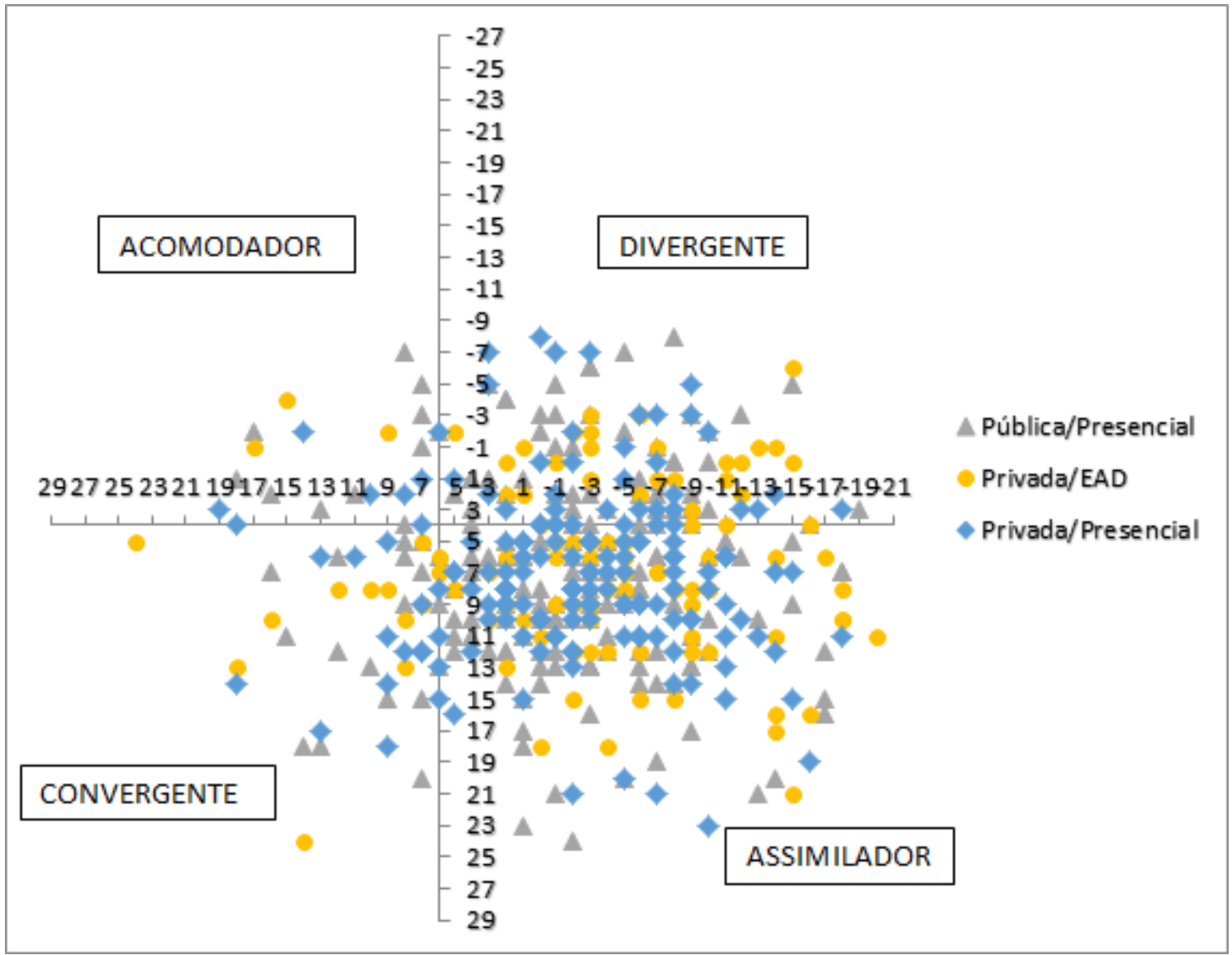

Gráfico 2 Distribuição dos Estilos de Aprendizagem.

Fonte: Elaboração própria (2015).

O gráfico 2 evidencia o estilo de aprendizagem assimilador como predominante entre o grupo pesquisado. O quadrante inferior direito apresenta uma distribuição uniforme entre as variáveis. Nota-se que o estilo divergente está em segundo lugar, pode-se verificar no quadrante superior direito. O estilo menos presente é o acomodador localizado no quadrante superior esquerdo.

Tabela 4 Estilo de Aprendizagem Predominante.

\begin{tabular}{l|c|c|c|c|c|c}
\hline \multirow{2}{*}{$\begin{array}{c}\text { ESTILO DE } \\
\text { APRENDIZAGEM }\end{array}$} & \multicolumn{2}{|c|}{ GÊNERO } & \multicolumn{2}{c|}{$\begin{array}{c}\text { TIPO DE } \\
\text { INSTITUIC̃O }\end{array}$} & $\begin{array}{c}\text { MODALIDADE DE } \\
\text { ENSINO }\end{array}$ \\
\cline { 2 - 7 } Convergente & Masculino & Feminino & Pública & Privada & Presencial & EAD \\
\hline Divergente & $8 \%$ & $8 \%$ & $8 \%$ & $9 \%$ & $8 \%$ & $9 \%$ \\
\hline Acomodador & $25 \%$ & $27 \%$ & $24 \%$ & $27 \%$ & $23 \%$ & $27 \%$ \\
\hline Assimilador & $5 \%$ & $5 \%$ & $6 \%$ & $4 \%$ & $6 \%$ & $4 \%$ \\
\hline Total & $62 \%$ & $60 \%$ & $62 \%$ & $61 \%$ & $63 \%$ & $55 \%$ \\
\hline
\end{tabular}

Fonte: Elaboração própria (2015). 
Pode-se concluir que, independente das varáveis: tipo de instituição, modalidade de ensino e gênero, os alunos participantes da pesquisa apresentam preferência assimiladora, correspondendo uma média de $60 \%$ da amostra. Os dados evidenciam uma intenção dos discentes a aprenderem mais com atividades concretas e lógicas.

Pelo fato dos estudantes apresentaram forte preferência pelo estilo assimilador, isso não significa que não podem desenvolver outros estilos ou até mesmo todos eles. Consoantes com esses resultados são os encontrados por Nogueira et al. (2012) o qual pesquisou 109 alunos do curso de administração na FEA/USP e identificou como estilo predominante o assimilador.

Cerqueira (2000) pesquisou 2550 universitários de várias regiões do Brasil, o estudo envolveu oito áreas do conhecimento, os alunos apresentaram estilo de aprendizagem assimilador. Pessoas com esse estilo captam as informações pela conceituação abstrata (CA) e a processam de forma reflexiva. Para Sobral (2012), destacam-se por ter um raciocino rápido e indutivo apreciam leitura e valorizam modelos teóricos e práticos. Manochehr (2006), Tanner e Morgan (2007) e Santos et al. (2013) encontraram resultados que o estilo Assimilador também apresenta melhores resultados.

Segundo Kolb (2005), os assimiladores têm habilidades para lidar com as ciências exatas. Partindo desse entendimento, conclui-se que os alunos do curso de Ciências Contábeis podem fazer essa opção, por que esse curso possui grande utilização de conteúdos numéricos. Dessa forma, verifica-se uma adequação do resultado desta pesquisa com o perfil exposto pela teoria da área contábil.

Os resultados apresentados são úteis, pois demonstram que embora o estilo de aprendizagem predominante entre as variáveis analisadas foi o assimilador, observa-se uma variação entre os quatro estilos, com o estilo divergente como segundo perfil mais destacado. Dessa forma, o docente de Contabilidade deve está ciente do exercício profissional e das diferenças de estilo de aprendizagem quando da aplicação de metodologia de ensino.

\section{CONSIDERAÇÕES FINAIS}

A partir da análise do instrumento de Kolb aplicado em 459 estudantes de graduação em Ciências Contábeis do Estado da Bahia, foi possível identificar o estilo de aprendizagem predominante nos alunos de instituições públicas e privadas nas modalidades de ensino presencial e EAD. 
Com relação ao problema da pesquisa, o qual consiste em analisar se existem diferenças de estilo de aprendizagem predominante em alunos do curso de Ciências Contábeis que estudam na modalidade de ensino presencial e a distância em instituição pública ou privada, constatou-se não haver diferenças entre as variáveis envolvidas, respondendo, assim, ao problema proposto.

A predominância do estilo assimilador mostrou-se estável em todas as turmas analisadas, independente de gênero, tipo de instituição e modalidade de ensino, sendo insignificantes as diferenças identificadas. Uma característica importante dos estudantes que possuem esse estilo é que à medida que vão perdendo características de pensar e observar sobre determinado assunto, vão adquirindo modos de aprendizagem apoiados no sentir e fazer, ou seja, na experiência concreta e na experimentação ativa.

Cabe ressaltar que os resultados do referido estudo restringem-se ao grupo pesquisado. Desta forma, não se pode afirmar que os achados representam todos os estudantes de Contabilidade do Estado da Bahia, contudo, são corroborados pelos achados de Cerqueira (2000), Manochehr (2006), Tanner e Morgan (2007), Nogueira et al. (2012), Reis, Paton e Nogueira (2012), Batista (2013) e Santos et al. (2013) no que se refere a estilo de aprendizagem predominante em alunos de Contabilidade.

Este estudo contribui para melhoria do ensino aprendizagem em Contabilidade, uma vez que possibilita ao professor conhecer o estilo de aprendizagem do aluno e assim, criar mecanismos cognitivos que facilitem o processamento das informações ao mesmo tempo em que permite direcionar sua metodologia levando em consideração o estilo predominante.

Novos estudos podem ser realizados com o mesmo instrumento utilizado nesta pesquisa para verificar a influência do estilo de aprendizagem no desempenho acadêmico, incluindo novas variáreis como aspectos motivacionais dos alunos e atividades profissionais exercidas.

\section{REFERÊNCIAS}

American Accounting Institute Of Certified Public Accountants (2000). Core Competency Framework for Entry into the Accounting Profession. USA.

Brasil. Decreto lei $n^{\circ} 15.601$ de 26 de janeiro de 1946. Dispõe sobre instalação da faculdade de Ciências Econômicas e Administração da Universidade de São Paulo. Disponível em: < http://www.al.sp.gov.br/norma/?id=66930> Acessado em 19 de fevereiro do ano de 2014.

Batista. C.T. (2013). ESTILOS DE APRENDIZAGEM E MÉTODOS DE ENSINO NO CURSO DE CIÊNCIAS CONTÁBEIS DO CAMPUS VII DA UNEB. 
Cerqueira, T. C. S (2000). Estilos de Aprendizagem em Universitários. 2000.179 f. Tese (Doutorado em Psicologia). Universidade Estadual de Campinas Faculdade de Educação. Campinas,. Disponível em: $<$ http://www.bibliotecadigital.unicamp.br/document/?code $=$ vtls000197620 $>$. Acesso em 10 de abril de 2014.

Cornachione Jr, E. B,; Casa Nova, Silvia Pereira De Castro; Trombetta, Maria Rosa (2007). Educação on-line em contabilidade: propensão e aspectos curriculares. Revista Contabilidade \& Finanças (Impresso), v. 18, p. 9-21.

Cox, T. D (2013). Learning Styles and Admission Criteria as Predictors of Academic Performance of College Freshmen. Institute for Learning Styles Journal Volume, 1. Disponível em: $<$ http://www.auburn.edu/academic/education $>$. Acesso em 01 de março de 2014.

Freire, Paulo (2007). Pedagogia da Autonomia: saberes necessários à prática educativa. 35 ed. São Paulo: Paz e Terra.

Lima, F. R. N., Bruni, A. L., \& Sampaio, M. S. (2012). A Influência do Gênero, Idade e Formação na Presença de Heurísticas em Decisões de Orçamento: Um Estudo QuaseExperimental. Revista Universo Contábil, 8 (2), 103-117.

Kolb, David A (1984). Experiential learning: experience as the source of learning and development. Englewood Cliffs, NJ (USA): Prentice-Hall.

Lima, A (2007). O Estilos de aprendizagem segundo os postulados de David Kolb: uma experiência no curso de odontologia da UNOESTE. Diss. Dissertação (Mestrado em Educação)-Departamento de Educação, Universidade do Oeste Paulista, Presidente Prudente.

Manochehr, Naser-Nick (2006). The influence of learning styles on learners in E-learning environments: an empirical study. Journal Computers in Higher Education Economics Review. Bristol, 18, 1, p. 10-14.

Manolis, Chris et al (2013). Assessing experiential learning styles: A methodological reconstruction and validation of the Kolb Learning Style Inventory. Learning and Individual Differences, v. 23, p. 44-52.

Mccarthy, Mary (2010). Experiential learning theory: from theory to practice. Journal of Business \& Economics Research, v. 8, n. 5.

Mcchlery, Stuart; Visser, Susan (2009). A comparative analysis of the learning styles of accounting students in the United Kingdom and South Africa. Registered Number: 1072954 Registered office: Mortimer House, 37-41, Mortimer Street, London W1T 3JH, UK.

Mill, D., Ribeiro, L. R. G., \& Oliveira, M. R. G (2010). Polidocência na educação a distância: múltiplos enfoques. São Carlos: EdUFSCar.

Moore, M. \& Kearsley, G (2007). Educação a distância: uma visão integrada. São Paulo: Thomson Learning. 
Nogueira, D. R (2009). O impacto do estilo de aprendizagem no desempenho acadêmico: um estudo empírico com alunos das disciplinas de Contabilidade geral e gerencial na Educação à distância. Dissertação (Mestrado em Contabilidade). Programa de Mestrado em Contabilidade do Setor de Ciências Sociais Aplicadas da Universidade Federal do Paraná.

Nogueira. R.D; Espejo B. S.M.M; Reis. G.L (2012). Estilos De Aprendizaje y Desempeño en Educación a Distancia: Un Estudio Empírico Con Alumnos de las Disciplinas de Contabilidad General y de Gestión. Revista de Educação e pesquisa em contabilidade. Brasilia, v, 6.n ${ }^{\mathrm{o}} 1$ art.4, p. 55-73.

Oliveira, Daniele Eufrásio de (2013). Impacto dos estilos de aprendizagem no desempenho acadêmico do ensino de contabilidade: uma análise dos estudantes da Universidade Federal do Rio Grande do Norte.

Oliveira, A. J.; Raffaelli, S. C. D.; Colauto, R. D.; Nova, S. P. C. C. (2013). Estilos de aprendizagem e estratégias ludopedagógicas: percepções no ensino da contabilidade. Advances in Scientific and Applied Accounting, v. 6, n. 2, p. 236-262.

Ku, David Tawei; Shih, Ju-Ling; Hung, Su-Huan (2014). The Integration of Concept Mapping in a Dynamic Assessment Model for Teaching and Learning Accounting. Educational Technology \& Society, v. 17, n. 1, p. 141-153.

Reis, L. G. dos, Paton, C., Nogueira, D. M (2012). Estilos de aprendizagem: uma análise dos alunos do curso de ciências contábeis pelo método Kolb. Enfoque: Reflexão Contábil, v. 31, n. 1, p. 53-66.

Richardson, Peter et al (2013). Students' perceptions on using iPods in accounting education: a mobile-learning experience. Asian Review of Accounting, v. 21, n. 1, p. 4-26.

Santos, C. A. D., Moraes, C. C. D., Rodrigues, C. B., \& Evangelista, L. D. A. (2014). Estilos De Aprendizagem: Um Estudo Empírico Com Alunos Do Curso De Ciências Contábeis Da Universidade Federal De Mato Grosso Do Sul. Revista Razão Contábil \& Finanças, 4(2).

Silva, D. M (2006). O Impacto dos Estilos de Aprendizagem no Ensino da Contabilidade da FEA-RP/USP. Dissertação (Mestrado) - Faculdade de Economia, Administração e Contabilidade. Universidade de São Paulo. Ribeirão Preto, 169 p

Sobral, Dejano T (2012). Inventário de estilo de aprendizagem de Kolb: Características e relação com resultados de avaliação no ensino pré-clínico. Psicologia: Teoria e Pesquisa, v. 8, n. 3, p. 293-303.

Tanner, Raquel Cristina Silva; Morgan (2007), Beatriz Fátima. Estilos de aprendizagem em universitários: uma análise sobre os alunos das disciplinas de contabilidade geral I e introdução à contabilidade na universidade de Brasília. In: Congresso USP de Controladoria e Contabilidade, $7^{\mathrm{o}}$.

Turesky, Elizabeth Fisher; Gallagher, Dennis (2011). Know thyself: Coaching for leadership using Kolb's experiential learning theory. The Coaching Psychologist, v. 7, n. 1, p. 5-14. 